\title{
Solution generating in scalar-tensor theories with a massless scalar field and stiff perfect fluid as a source
}

\author{
Stoytcho S. Yazadjiev \\ Department of Theoretical Physics, Faculty of Physics, Sofia University, \\ 5 James Bourchier Boulevard, 1164 Sofia, Bulgaria \\ E-mail: yazad@phys.uni-sofia.bg
}

\begin{abstract}
We present a method for generating solutions in some scalar-tensor theories with a minimally coupled massless scalar field or irrotational stiff perfect fluid as a source. The method is based on the group of symmetries of the dilaton-matter sector in the Einstein frame. In the case of Barker's theory the dilaton-matter sector possesses $S U(2)$ group of symmetries. In the case of Brans-Dicke and the theory with "conformal coupling", the dilaton- matter sector has $S L(2, R)$ as a group of symmetries. We describe an explicit algorithm for generating exact scalar-tensor solutions from solutions of Einstein-minimally-coupled-scalar-field equations by employing the nonlinear action of the symmetry group of the dilaton-matter sector. In the general case, when the Einstein frame dilaton-matter sector may not possess nontrivial symmetries we also present a solution generating technique which allows us to construct exact scalar-tensor solutions starting with the solutions of Einstein-minimally-coupled-scalar-field equations. As an illustration of the general techniques, examples of explicit exact solutions are constructed. In particular, we construct inhomogeneous cosmological scalar-tensor solutions whose curvature invariants are everywhere regular in spacetime. A generalization of the method for scalar-tensor-Maxwell gravity is outlined.
\end{abstract}

PACS numbers: 04.50.+h, 04.20.Jb, 98.80.Hw

\section{INTRODUCTION}

Scalar-tensor theories of gravity are considered as the most natural generalizations of general relativity [1]- [6]. In these theories gravity is mediated not only by the metric of space-time but also by a scalar field (the so called gravitational scalar). Scalar-tensor theories contain arbitrary functions of the scalar field that determine the gravitational "constant" as a dynamical variable. From a theoretical point of view it should be noted that specific scalar-tensor theories arise naturally as a low-energy limit of string theory.

In the weak field limit scalar-tensor theories differ slightly from general relativity. In the strong field regime, however, the predictions of scalar-tensor theories may differ drastically from those of general relativity as it was shown in Refs. 77 and [8].

Scalar-tensor theories have also attracted much interest in cosmology (see Refs. [9]- 27 and references therein).

The progress in the understanding of scalar-tensor theories of gravity is closely connected with finding and investigation of exact solutions. A theoretical discussions of many aspects of the early universe, gravitational waves, gravitational collapse and the structure of the compact objects within the framework of scalar-tensor theories (as in general relativity) necessitates the use of exact solutions. Besides the theoretical motivation for construction of exact solutions, there is also a more practical one. With the advent of numerical calculations, exact solutions are useful as comparisons for numerical and approximate solutions and as checks of the computer codes.

Solving of scalar-tensor theories equations in the presence of a source is a difficult task due to their complexity in the general case. In fact, scalar-tensor gravity equations are much more complicated than Einstein equations. In the so called Einstein frame the sourceless scalar-tensor equations are reduced to Einstein equations with a minimally coupled scalar field. In this case much progress has been achieved in finding exact homogeneous and inhomogeneous cosomological solutions [28][37. Little has been done in solving scalar-tensor equations with a source. The known solutions are perfect fluid homogeneous cosmological solutions depending on the time coordinate only (see Refs. [12]- [20] and references therein). In Ref. [12], Barrow investigated a method which enables exact solutions to be found for vacuum and radiation dominated Friedmann-RobertsonWalker (FRW) universes of all curvatures in scalar-tensor theories with an arbitrary form of the coupling function $\omega(\Phi)$. Particular classes of solutions were presented for specific choices of $\omega(\Phi)$, including Brans-Dicke, Barker and Bekenstein theories. Barrow and Mimoso [13] presented a method for deriving exact solutions for flat FRW cosmological models with a perfect fluid satisfying the equation of state $p=(\gamma-1) \rho$ where $\gamma$ is constant with $0 \leq \gamma \leq 2$, in scalar-tensor theories with an arbitrary form for the coupling function $\omega(\Phi)$. A number of explicit solutions for inflationary universes and $p=0$ uni- 
verses were obtained. Exact FRW cosmological solutions in general scalar-tensor theories for stiff perfect fluid or radiation were derived by Mimoso and Wands in Ref. [14]. Homogeneous but anisotropic cosmologies in scalartensor theories of gravity were examined by Mimiso and Wands in Ref. [15]. The authors presented a method for deriving solutions for any isotropic perfect fluid with a barotropic equation of state in a spacially flat cosmology. For stiff fluid or radiation or in vacuum the authors were able to obtain solutions in a number of anisotropic Bianchi and Kantowski-Sachs metrics. Extending the earlier work of [11], [12] and [13, Barrow and Parsons [16] provided a detailed analysis of FRW universes in a wide range of scalar-tensor theories of gravity. The authors constructed a range of exact solutions for open, closed and flat isotropic universes containing matter with an equation of state $p \leq(1 / 3) \rho$ and in vacuum. The early and late-time behaviours of the solutions were examined, too. In Ref. [17], Billyard and Coley discussed the formal equivalences between Kaluza-Klein gravity, Brans-Dicke theory and general relativity coupled to a massless scalar field. Using the formal equivalences the authors showed that exact solutions obtained in one theory will correspond to analogous solutions in the other two theories. A phase-space analysis of the FRW models in Brans-Dicke theory was performed by Kolitch and Eardley [18]. Their analysis was improved on by Holden and Wands [19] who presented all FRW models in a single phase plane. Particular attention was focused on the early and late-time behaviour of the solutions and on whether inflation occurs. The qualitative properties of scalar-tensor theories of gravity were also studied by Coley in Ref. [20]. The author presented exact solutions which are analogues of the general relativistic Jacobs stiff perfect fluid solutions and vacuum plane wave solutions which act as past and future attractors in the class of spatially homogeneous models in Brans-Dicke theory.

It should be noted the the methods developed in [12], [13], [14], and [15] are solution generating methods only for homogeneous cosmological solutions and are not applicable to more general cases.

The purpose of this paper is to present a general method for generating exact solutions to the gravity equations with a minimally coupled massless scalar field (MCSF) and irrotational stiff perfect fluid within the framework of some scalar-tensor theories whose Einstein frame dilaton-matter sector has nontrivial symmetries. In the general case, when the Einstein frame dilatonmatter sector may not possess nontrivial symmetries we also present a general solution generating technique which allows us to construct exact scalar-tensor solutions starting with the solutions of Einstein-minimallycoupled-scalar-field (EMCSF). This technique is based on the geodesics of the Riemannian metric associated with the dilaton-matter sector.

The motivations to consider a MCSF as a source are the following. In view of the complexity of the equations of the scalar-tensor gravity, it is natural as a first step to consider a simple source. On the other hand, in different contexts, the scalar field (different from the gravitational scalar) plays an important role in modern physics: the scalar field has been proposed as a candidate for gravitational lensing [38], [39] and for dark matter at galaxies scales [40], as well as at cosmological scales [41], 442], 433.

Examples of different kinds of explicit exact solutions are also considered.

\section{SCALAR-TENSOR THEORIES WITH A MINIMALLY COUPLED SCALAR FIELD AS A SOURCE AND SYMMETRIES OF DILATON - MATTER SECTOR}

Scalar-tensor theories are described by the following action in the Jordan frame:

$$
\begin{aligned}
& S=\frac{1}{16 \pi G_{*}} \int d^{4} x \sqrt{-\tilde{g}}\left(F(\Phi) \tilde{R}-Z(\Phi) \tilde{g}^{\mu \nu} \partial_{\mu} \Phi \partial_{\nu} \Phi\right. \\
& -2 U(\Phi))+S_{m}\left[\Psi_{m} ; \tilde{g}_{\mu \nu}\right] .
\end{aligned}
$$

Here, $G_{*}$ is the bare gravitational constant, $\tilde{R}$ is the Ricci scalar curvature with respect to the space-time metric $\tilde{g}_{\mu \nu}$. The dynamics of the scalar field $\Phi$ depends on the functions $F(\Phi), Z(\Phi)$ and $U(\Phi)$. In order for the gravitons to carry positive energy the function $F(\Phi)$ must be positive. The action of matter depends on the material fields $\Psi_{m}$ and the space-time metric $\tilde{g}_{\mu \nu}$ but does not involve the scalar field $\Phi$ in order for the weak equivalence principle to be satisfied. It should be mentioned that the most used parametrization in the literature is Brans-Dicke one, corresponding to $F(\Phi)=\Phi$ and $Z(\Phi)=\omega(\Phi) / \Phi$.

It is much clearer to analyze the equations in the socalled Einstein frame. Let us introduce the new variables $g_{\mu \nu}$ and $\varphi$, and define

$$
\begin{array}{r}
g_{\mu \nu}=F(\Phi) \tilde{g}_{\mu \nu} \\
\left(\frac{d \varphi}{d \Phi}\right)^{2}=\frac{3}{4}\left(\frac{d \ln (F(\Phi))}{d \Phi}\right)^{2}+\frac{Z(\Phi)}{2 F(\Phi)} \\
\mathcal{A}(\varphi)=F^{-1 / 2}(\Phi) \\
2 V(\varphi)=U(\Phi) F^{-2}(\Phi)
\end{array}
$$

From now on we will refer to $g_{\mu \nu}$ and $\varphi$ as Einstein frame metric and dilaton field correspondingly.

In the Einstein frame the action (11) takes the form

$$
\begin{array}{r}
S=\frac{1}{16 \pi G_{*}} \int d^{4} x \sqrt{-g}\left(R-2 g^{\mu \nu} \partial_{\mu} \varphi \partial_{\nu} \varphi-4 V(\varphi)\right) \\
+S_{m}\left[\Psi_{m} ; \mathcal{A}^{2}(\varphi) g_{\mu \nu}\right]
\end{array}
$$

where $R$ is the Ricci scalar curvature with respect to the Einstein frame metric $g_{\mu \nu}$.

We consider scalar-tensor theories with a minimally coupled (massless) scalar filed (MCSF), $\sigma$, as a matter source. A minimally coupled massless scalar field also 
corresponds to an irrotational stiff perfect fluid with energy density

$$
8 \pi G_{*} \tilde{\rho}=8 \pi G_{*} \tilde{p}=-\tilde{g}^{\mu \nu} \partial_{\mu} \sigma \partial_{\nu} \sigma
$$

and velocity field

$$
\tilde{u}_{\mu}=\partial_{\mu} \sigma / \sqrt{-\tilde{g}^{\mu \nu} \partial_{\mu} \sigma \partial_{\nu} \sigma}
$$

provided $\tilde{g}^{\mu \nu} \partial_{\mu} \sigma \partial_{\nu} \sigma<0$ [36], 37].

The Jordan frame action for the scalar field is

$$
S_{m}=\frac{1}{16 \pi G_{*}} \int d^{4} x \sqrt{-\tilde{g}}\left(-2 \tilde{g}^{\mu \nu} \partial_{\mu} \sigma \partial_{\nu} \sigma\right) .
$$

In what follows we will consider only the special form of the potential $U(\Phi): U(\Phi)=2 \Lambda F^{2}(\Phi)$ or $V(\varphi)=\Lambda=$ const.

The full Einstein frame action then is

$$
S=\frac{1}{16 \pi G_{*}} \int d^{4} x \sqrt{-g}\left(R-\Lambda+\mathcal{L}_{D M}\right)
$$

where

$$
\mathcal{L}_{D M}=-2 g^{\mu \nu} \partial_{\mu} \varphi \partial_{\nu} \varphi-2 \mathcal{A}^{2}(\varphi) g^{\mu \nu} \partial_{\mu} \sigma \partial_{\nu} \sigma
$$

is the dilaton-matter sector of the theory Lagrangian.

Remarkably, in the case of some specific scalar-tensor theories the dilaton matter-sector (8) of the theory possesses hidden symmetries which allow us to generate exact solutions. In order to unveil these symmetries we define a two-dimensional abstract Riemannian space with a metric

$$
d l^{2}=d \varphi^{2}+\mathcal{A}^{2}(\varphi) d \sigma^{2} .
$$

Solution generation techniques consist in finding invariant transformations of the dilaton-matter sector of the Lagrangian in the action (7). This is equivalent to finding the isometry group of the metric (9). In two dimensions the isometry group of (9) can be either $G_{1}$ or $G_{3}$. Clearly our metric possesses $G_{1}$ isometry corresponding to the Killing vector $\partial / \partial \sigma$. From a physical point of view, however, this symmetry is not interesting because it generates just a shift of the scalar field $\sigma$ : $\sigma \rightarrow \sigma+$ const. The metric (9) has $G_{3}$ group of isometries only when the Gauss curvature $K$ is constant. Reversely, the constant curvature condition imposes a differential equation for the function $\mathcal{A}(\varphi)$ :

$$
K=-\mathcal{A}^{-1}(\varphi) \frac{d^{2} \mathcal{A}(\varphi)}{d \varphi^{2}} .
$$

In this way, solving the diferential equation (10) we obtain the scalar-tensor theories whose dilaton-matter sector possesses group of isometries $G_{3}$.

When the Gauss curvature is positive $(K>0)$ the group of isometries is $S U(2)$ (see below). The scalartensor theories corresponding to this case are characterized by

$$
\mathcal{A}(\varphi)=a \cos (\sqrt{K} \varphi+b)
$$

which corresponds to the functions $F(\Phi)=\Phi$ and

$$
Z(\Phi)=\frac{(1+3 K)-3 K a^{2} \Phi}{2 K \Phi\left(a^{2} \Phi-1\right)}
$$

where $a>0$ and $b$ are arbitrary constants. Since eq.(2) define $\varphi$ up to a constant we may put $b=0$. In the case $K=1$ and $a=1$ we obtain the Barker's theory [44].

For negative Gauss curvature $(K<0)$ the isometry group is $S L(2, R)$. The scalar-tensor theories whose dilaton-matter sector has negative curvature are characterized by

$$
\mathcal{A}(\varphi)=a \exp (\sqrt{|K|} \varphi)+b \exp (-\sqrt{|K|} \varphi)
$$

corresponding to the functions $F(\Phi)=\Phi$ and

$$
Z(\Phi)=\frac{(1-3|K|)+12|K| a b \Phi}{2|K| \Phi(1-4 a b \Phi)}
$$

where $a>0$ and $b \geq 0$ are arbitrary constants. In the case $b=0$ we obtain the Brans-Dicke theory with $|K|=1 /(3+2 \omega)$. When $a b \neq 0$ the function $\mathcal{A}(\varphi)$ can be presented in the form

$$
\mathcal{A}(\varphi)=\Omega \cosh (\sqrt{|K|} \varphi+c)
$$

where $\Omega=2 \sqrt{a b}$ and $c$ is a constant. Without loss of generality we may put $c=0$. In the special case $\Omega=1$ and $|K|=1 / 3$ we obtain the theory with "conformal coupling". This theory is also described by the functions $F(\Phi)=1-\frac{1}{6} \Phi^{2}$ and $Z(\Phi)=1$.

The scalar-tensor theories possessing flat $(K=0)$ dilaton-matter sector have an isometry group $I s o\left(\mathbf{R}^{2}\right)$ and are described by

$$
\mathcal{A}(\varphi)=1+a \varphi
$$

corresponding to the functions $F(\Phi)=\Phi$ and

$$
Z(\Phi)=\frac{1-3 a^{2} \Phi}{2 a^{2} \Phi^{2}}
$$

where $a \neq 0$ is an arbitrary constant.

It is worth noting that he scalar-tensor theories with the same group of isometries of the dilaton-matter sector may have rather different behaviour from a physical point of view. As for example we may consider the Barker's theory with $\mathcal{A}(\varphi) \sim \cos (\varphi)$ and a theory with $\mathcal{A}(\varphi) \sim \cos (\sqrt{K} \varphi)$ where $K \neq 1$. For the Barker's theory the effective gravitational "constant" is a real constant $G_{\text {eff }} \sim G_{*}$ (to first order of the weak filed limit) while for a theory with $K \neq 1$ the effective gravitational "constant" may vary : $G_{e f f} \sim \cos ^{2}(\sqrt{K} \varphi)+K \sin ^{2}(\sqrt{K} \varphi)$.

${ }^{*}$ Here we consider the case $\omega>-3 / 2$.

${ }^{\dagger}$ We have put the second constant in $\mathcal{A}(\varphi)$ equal to one. 
Below we consider in details the symmetries of the dilaton-matter sector for the scalar-tensor theories described above and present solutions generating formulas. The general case when dilaton-matter sector does not possess nontrivial symmetries is also considered.

\section{A. Barker's theory}

Barker's theory 44 is described by the functions $F(\Phi)=\Phi$ and $Z(\Phi)=(4-3 \Phi) / \Phi(2 \Phi-2)$ corresponding to $\mathcal{A}^{2}(\varphi)=\cos ^{2}(\varphi)$. The metric (9) then is

$$
d l^{2}=d \varphi^{2}+\cos ^{2}(\varphi) d \sigma^{2} .
$$

This metric can be considered as the standard metric on the unit 2-sphere. It is more convenient to present the metric in the well known complex form. To do so we introduce the complex field

$$
z=\cot (\varphi / 2+\pi / 4) e^{i \sigma}
$$

We obtain then

$$
d l^{2}=4 \frac{d z d \bar{z}}{(1+z \bar{z})^{2}}
$$

The metric is invariant under the transformations

$$
z \rightarrow z^{\prime}=\frac{a z+b}{-\bar{b} z+\bar{a}}
$$

where

$$
U=\left(\begin{array}{cc}
a & b \\
-\bar{b} & \bar{a}
\end{array}\right) \in S U(2) .
$$

There is also an independent discrete symmetry $z \rightarrow \bar{z}$ which corresponds to $\sigma \rightarrow-\sigma$.

We note that the $S U(2)$ transformations act nonlinearly on the scalar fields but leave the Einstein frame metric invariant: $g_{\mu \nu}^{\prime}=g_{\mu \nu}$. The Jordan frame metric $\tilde{g}_{\mu \nu}$, therefore, transforms under (14) as

$$
\tilde{g}_{\mu \nu}^{\prime} \Phi^{\prime}=\tilde{g}_{\mu \nu} \Phi
$$

The symmetries of the dilaton-matter sector can be used to generate new solutions from known ones. In the case of Barker's theory, any solution of Einstein equations with a minimally coupled scalar field (EMCSF) and cosmological term will be a solution of the Einstein frame scalar-tensor-MCSF equations with cosmological term and with vanishing $\varphi$ or $\sigma$. Therefore, we can construct Barker counterparts to any solution of the EMCSF equations using the nonlinear action of the group $S U(2)$.

An arbitrary element $U \in S U(2)$ can be presented in the form

$$
U=\mathcal{D}(\delta) \mathcal{O}(\beta) \mathcal{D}(\gamma)
$$

where

$$
\mathcal{D}(\gamma)=\left(\begin{array}{cc}
e^{i \gamma / 2} & 0 \\
0 & e^{-i \gamma / 2}
\end{array}\right)
$$

and

$$
\mathcal{O}(\beta)=\left(\begin{array}{cc}
\cos (\beta / 2) & \sin (\beta / 2) \\
-\sin (\beta / 2) & \cos (\beta / 2)
\end{array}\right) \in S O(2) .
$$

It should be noted that the transformations (14) associated with the subgroup consisting of the matrices $\mathcal{D}$ correspond to a constant shift of the scalar field $\sigma$ : $\sigma \rightarrow \sigma+$ const. That is why, without loss of generality, we may restrict ourselves to the subgroup $S O(2)$.

Taking as a seed solution $z_{0}=e^{i \sigma_{0}}$ and performing a $S O(2)$ transformation with the matrix $\mathcal{O}(\beta)$ we obtain a new solution $z$ which in terms of $\varphi$ and $\sigma$ reads

$$
\varphi=\arcsin \left[\sin (\beta) \cos \left(\sigma_{0}\right)\right]
$$

$$
\sigma=\arcsin \left[\frac{\sin \left(\sigma_{0}\right)}{\sqrt{1-\sin ^{2}(\beta) \cos ^{2}\left(\sigma_{0}\right)}}\right] .
$$

Once having the solution in the Einstein frame it is easy to recover the corresponding Jordan frame one. The results we summarize in the following proposition:

Proposition: Let $\left(g_{\mu \nu}, \sigma_{0}\right)$ be a solution to the EMCSF equations with a cosmological term $\Lambda$. Then $\left(\tilde{g}_{\mu \nu}, \Phi, \sigma\right)$ form a solution to the Barker-MCSF equations with a cosmological potential $U(\Phi)=2 \Lambda \Phi^{2}$ where $\sigma$ is given by (19) and

$$
\begin{gathered}
\Phi^{-1}\left(\sigma_{0}\right)=1-\sin ^{2}(\beta) \cos ^{2}\left(\sigma_{0}\right), \\
\tilde{g}_{\mu \nu}=\Phi^{-1}\left(\sigma_{0}\right) g_{\mu \nu} .
\end{gathered}
$$

Without going into detail we formulate the proposition in terms of a perfect fluid:

Proposition: Let $\left(g_{\mu \nu}, \rho_{0}, u_{\mu}^{(0)}\right)$ be a solution to the Einstein equations with an irrotational stiff perfect fluid and a cosmological term $\Lambda$. Then $\left(\tilde{g}_{\mu \nu}, \Phi, \tilde{\rho}, \tilde{u}_{\mu}\right)$ is a solution to the Barker's equations with an irrotational stiff perfect fluid and cosmological potential $U(\Phi)=2 \Lambda \Phi^{2}$ if $\Phi$ and $\tilde{g}_{\mu \nu}$ are given by (20) and (21), and

$$
\begin{aligned}
\tilde{\rho} & =\cos ^{2}(\beta) \Phi^{3}\left(\sigma_{0}\right) \rho_{0}, \\
\tilde{u}_{\mu} & =\Phi^{-1 / 2}\left(\sigma_{0}\right) u_{\mu}^{(0)}, \\
8 \pi G_{*} \rho_{0} & =-g^{\mu \nu} \partial_{\mu} \sigma_{0} \partial_{\nu} \sigma_{0} .
\end{aligned}
$$

\section{B. Brans-Dicke theory}

Brans-Dicke theory is characterized by the functions $F(\Phi)=\Phi$ and $Z(\Phi)=\omega / \Phi$ which correspond to 
$\mathcal{A}^{2}(\varphi)=e^{2 \alpha \varphi}$ where $\alpha^{2}=|K|=1 /(3+2 \omega)$. In the Brans-Dicke case the metric (9) takes the form

$$
d l^{2}=d \varphi^{2}+e^{2 \alpha \varphi} d \sigma^{2} .
$$

The metric (23), up to the parameter $\alpha$, also appears in the so called dilaton-axion gravity [45]. It should be noted, however, that we consider (23) in a different physical context. The symmetries of dilaton-axion sector are well known, but for completeness we will describe them again in our context.

Introducing the complex field

$$
\xi=\alpha \sigma+i e^{-\alpha \varphi},
$$

we can write the metric (23) in the Klein form

$$
d l^{2}=-\frac{4}{\alpha^{2}} \frac{d \xi d \bar{\xi}}{(\xi-\bar{\xi})^{2}} .
$$

The dilaton-matter sector is $S L(2, R)$ invariant:

$$
\xi \rightarrow \xi^{\prime}=\frac{A \xi+B}{C \xi+D}
$$

where

$$
L=\left(\begin{array}{cc}
A & B \\
C & D
\end{array}\right) \in S L(2, R)
$$

There is also a discrete symmetry $\xi \rightarrow-\bar{\xi}$ which corresponds to $\sigma \rightarrow-\sigma$.

The Jordan frame metric $\tilde{g}_{\mu \nu}$ transforms under (26) as $\tilde{g}_{\mu \nu}^{\prime} \Phi^{\prime}=\tilde{g}_{\mu \nu} \Phi$.

Let us consider a solution $\left(g_{\mu \nu}, \sigma_{0}\right)$ to the EMCSF equations. This solution is also a solution to the Einstein frame scalar-tensor equations with $\xi_{0}=i e^{-\alpha \sigma_{0}}$. The $S L(2, R)$ transformation with the matrix $L$ gives a new solution $z=\alpha \sigma+i e^{-\alpha \varphi}$ where

$$
\begin{gathered}
e^{\alpha \varphi}=C^{2} e^{-\alpha \sigma_{0}}+D^{2} e^{\alpha \sigma_{0}}, \\
\sigma=\frac{1}{\alpha} \frac{A C e^{-\alpha \sigma_{0}}+B D e^{\alpha \sigma_{0}}}{C^{2} e^{-\alpha \sigma_{0}}+D^{2} e^{\alpha \sigma_{0}}} .
\end{gathered}
$$

In this way we proved the following proposition:

Proposition: Let $\left(g_{\mu \nu}, \sigma_{0}\right)$ be a solution to the EMCSF equations with a cosmological term $\Lambda$. Then $\left(\tilde{g}_{\mu \nu}, \Phi, \sigma\right)$ form a solution to the Brans-Dicke equations with a MCSF and a cosmological potential $U(\Phi)=2 \Lambda \Phi^{2}$ where $\sigma$ is given by (28) and

$$
\begin{aligned}
\Phi^{-1 / 2}\left(\sigma_{0}\right) & =C^{2} e^{-\alpha \sigma_{0}}+D^{2} e^{\alpha \sigma_{0}}, \\
\tilde{g}_{\mu \nu} & =\Phi^{-1}\left(\sigma_{0}\right) g_{\mu \nu} .
\end{aligned}
$$

Applied to the case of a stiff perfect fluid the proposition becomes:
Proposition: Let $\left(g_{\mu \nu}, \rho_{0}, u_{\mu}^{(0)}\right)$ be a solution to the Einstein equations with an irrotational stiff perfect fluid and a cosmological term $\Lambda$. Then $\left(\tilde{g}_{\mu \nu}, \Phi, \tilde{\rho}, \tilde{u}_{\mu}\right)$ is a solution to the Brans-Dicke equations with an irrotational stiff perfect fluid and a cosmological potential $U(\Phi)=$ $2 \Lambda \Phi^{2}$ if $\Phi$ and $\tilde{g}_{\mu \nu}$ are given by (29) and (39), and

$$
\begin{aligned}
\tilde{\rho} & =4 C^{2} D^{2} \Phi^{3}\left(\sigma_{0}\right) \rho_{0}, \\
\tilde{u}_{\mu} & =\Phi^{-1 / 2}\left(\sigma_{0}\right) u_{\mu}^{0}, \\
8 \pi G_{*} \rho_{0} & =-g^{\mu \nu} \partial_{\mu} \sigma_{0} \partial_{\nu} \sigma_{0} .
\end{aligned}
$$

The transformations (26) associated with the subgroup consisting of matrices of the form

$$
\mathcal{T}=\left(\begin{array}{cc}
A & B \\
0 & A^{-1}
\end{array}\right)
$$

correspond to either a constant shift or rescaling the scalar-fields. That is why, without loss of generality, we may restrict ourselves to the matrices

$$
\mathcal{O}(\beta)=\left(\begin{array}{cc}
\cos (\beta / 2) & \sin (\beta / 2) \\
-\sin (\beta / 2) & \cos (\beta / 2)
\end{array}\right) \in S O(2) .
$$

$$
\text { C. } \mathcal{A}(\varphi) \sim \cosh (\sqrt{|K|} \varphi) \text { theory }
$$

Here we consider the scalar-tensor theories with $\mathcal{A}(\varphi)=\Omega \cosh (\sqrt{|K|} \varphi), \Omega>0$. Introducing the complex field

$$
\zeta=\exp (\Omega \sqrt{|K|} \sigma)\left(\tanh (\sqrt{|K|} \varphi)+\frac{i}{\cosh (|K| \varphi)}\right)
$$

the metric can be written in the Klein form

$$
d l^{2}=-\frac{4}{|K|} \frac{d \zeta d \bar{\zeta}}{(\zeta-\bar{\zeta})^{2}} .
$$

The case under consideration is analogous to the BranDicke case and we present directly the final results omitting the intermediate details:

Proposition: Let $\left(g_{\mu \nu}, \sigma_{0}\right)$ be a solution to the EMCSF equations with a cosmological term $\Lambda$. Then $\left(\tilde{g}_{\mu \nu}, \Phi, \sigma\right)$ form a solution to the equations of the $\mathcal{A}(\varphi)=$ $\Omega \cosh (\sqrt{|K|} \varphi)$ theory with a MCSF and a cosmological potential $U(\Phi)=2 \Lambda \Phi^{2}$ if $\sigma, \Phi$ and $\tilde{g}_{\mu \nu}$ are given by

$$
\begin{aligned}
& e^{2 \Omega \sqrt{|K|} \sigma}=\frac{1+\left(A C e^{\sqrt{|K|} \sigma_{0}}+B D e^{-\sqrt{|K|} \sigma_{0}}\right)^{2}}{\left(C^{2} e^{\sqrt{|K|} \sigma_{0}}+D^{2} e^{-\sqrt{|K|} \sigma_{0}}\right)^{2}}, \\
& \Phi^{-1}\left(\sigma_{0}\right)=\Omega^{2}\left[1+\left(A C e^{\sqrt{|K|} \sigma_{0}}+B D e^{-\sqrt{|K|} \sigma_{0}}\right)^{2}\right],
\end{aligned}
$$




$$
\tilde{g}_{\mu \nu}=\Phi^{-1}\left(\sigma_{0}\right) g_{\mu \nu} .
$$

It is not difficult to rewrite this proposition for the case of a stiff perfect fluid. The fluid energy density is given by

$$
\tilde{\rho}=\Omega^{2}(A D+B C)^{2} \Phi^{3}\left(\sigma_{0}\right) \rho_{0} .
$$

\section{Theory with a flat dilaton-matter sector}

As we have seen, the theory with a flat dilaton-matter sector is described by $\mathcal{A}(\varphi)=1+a \varphi$. Let us introduce the complex field

$$
\varsigma=(1+a \varphi) e^{i \sigma} .
$$

The dilaton-matter sector metric then takes the standard flat form

$$
d l^{2}=d \varsigma d \bar{\varsigma}
$$

The metric is invariant under the group $I s o\left(\mathbf{R}^{2}\right)$ i.e. under the transformations

$$
\varsigma \rightarrow \varsigma^{\prime}=e^{i \vartheta} \varsigma+\eta
$$

where $\vartheta \in \mathbf{R}$ and $\eta=A+i B \in \mathbf{C}$.

Let us take as a seed solution $\varsigma_{0}=1+a \sigma_{0}$. It is not difficult to see that two of the parameters of the group Iso $\left(\mathbf{R}^{2}\right)$ can be absorbed as constant shifts $\sigma \rightarrow \sigma+$ const, and only one essential parameter remains. Performing a translation with $\eta=i B$ we obtain a new solution $\varsigma$ with

$$
\begin{gathered}
(1+a \varphi)=\sqrt{\left(1+a \sigma_{0}\right)^{2}+B^{2}}, \\
\sigma=\frac{1}{a} \arcsin \left[\frac{B}{\sqrt{\left(1+a \sigma_{0}\right)^{2}+B^{2}}}\right]
\end{gathered}
$$

So we have:

Proposition: Let $\left(g_{\mu \nu}, \sigma_{0}\right)$ be a solution to the EMCSF equations with a cosmological term $\Lambda$. Then $\left(\tilde{g}_{\mu \nu}, \Phi, \sigma\right)$ form a solution to the equations of the $\mathcal{A}(\varphi)=$ $1+a \varphi$ theory with a MCSF and a cosmological potential $U(\Phi)=2 \Lambda \Phi^{2}$ if $\sigma$ is given by $(43)$ and

$$
\begin{gathered}
\Phi^{-1}\left(\sigma_{0}\right)=\left(1+a \sigma_{0}\right)^{2}+B^{2}, \\
\tilde{g}_{\mu \nu}=\Phi^{-1}\left(\sigma_{0}\right) g_{\mu \nu} .
\end{gathered}
$$

In the case of a stiff perfect fluid we obtain

$$
\tilde{\rho}=B^{2} \Phi^{3}\left(\sigma_{0}\right) \rho_{0} .
$$

\section{E. Other theories}

When the metric (9) has nontrivial isometries we can use them to generate new solutions from known ones. In the general case, however, the metric (9) has only trivial symmetry $\sigma \longrightarrow \sigma+$ constant. That is why the solution generating procedure considered above fails. Nevertheless, when the dilaton-matter sector does not possess nontrivial symmetries we can still generate scalar-tensor solutions starting with solutions to the EMCSF equations. For this purpose we consider the geodesics of the metric (9). It is not difficult to see that if $(\varphi(\tau), \sigma(\tau))$ is an affinely parameterized geodesic and $\left(g_{\mu \nu}, \sigma_{0}\right)$ is a solution to the EMCSF equations with a cosmological term $\Lambda$, then $\left(g_{\mu \nu},\left.\varphi(\tau)\right|_{\tau=\sigma_{0}},\left.\sigma(\tau)\right|_{\tau=\sigma_{0}}\right)$ is a solution to the scalar-tensor-MCSF equations with a cosmological term $\Lambda$.

In this way we obtain the following

Proposition: Let $(\varphi(\tau), \sigma(\tau))$ is an affinely parameterized geodesic of the metric (9) and $\left(g_{\mu \nu}, \sigma_{0}\right)$ is a solution to the EMCSF equations with a cosmological term $\Lambda$. Then $\left(\tilde{g}_{\mu \nu}, \Phi, \sigma\right)$ form a solution to the scalartensor equations with a MCSF and a cosmological potential $U(\Phi)=2 \Lambda F^{2}(\Phi)$ where

$$
\begin{gathered}
\sigma=\left.\sigma(\tau)\right|_{\tau=\sigma_{0}}, \\
F^{-1}\left(\Phi\left(\sigma_{0}\right)\right)=\left.\mathcal{A}^{2}(\varphi(\tau))\right|_{\tau=\sigma_{0}}, \\
\tilde{g}_{\mu \nu}=F^{-1}\left(\Phi\left(\sigma_{0}\right)\right) g_{\mu \nu} .
\end{gathered}
$$

The geodesic equations for the metric (9) can be formally solved and we obtain

$$
\begin{array}{r}
\int \frac{d \varphi}{\sqrt{1-C^{2} / \mathcal{A}^{2}(\varphi)}}=C_{1} \pm \tau, \\
\sigma=C_{2}+C \int \frac{d \tau}{\mathcal{A}^{2}(\varphi(\tau))}
\end{array}
$$

where $C, C_{1}$ and $C_{2}$ are constants. The constants $C_{1}$ and $C_{2}$ are unimportant and we shall omit them.

As a concrete example we consider the theory with $F(\Phi)=\Phi$ and $Z(\Phi)=\frac{1}{2} \frac{\Phi^{2}-3 \Phi+3}{\Phi(\Phi-1)}$ corresponding to $\mathcal{A}^{2}(\varphi)=1 /\left(1+\varphi^{2}\right)$. Solving the geodesic equations for the metric $d l^{2}=d \varphi^{2}+\frac{d \sigma^{2}}{1+\varphi^{2}}$ we obtain

$$
\begin{gathered}
\varphi=\frac{1}{C} \sqrt{1-C^{2}} \sin \left(C \sigma_{0}\right), \\
\sigma=\frac{1+C^{2}}{2 C} \sigma_{0}-\frac{1-C^{2}}{4 C^{2}} \sin \left(2 C \sigma_{0}\right)
\end{gathered}
$$

where $0<C^{2}<1$.

Therefore, if $\left(g_{\mu \nu}, \sigma_{0}\right)$ is a solution to the EMCSF equations with a cosmological term $\Lambda$, then $\left(\tilde{g}_{\mu \nu}, \Phi, \sigma\right)$ is a solution to the equations of $\mathcal{A}^{2}(\varphi)=1 /\left(1+\varphi^{2}\right)$-theory 
with a MCSF and a cosmological potential $U(\Phi)=2 \Lambda \Phi^{2}$ where $\sigma$ is given by (52) and

$$
\begin{aligned}
\Phi\left(\sigma_{0}\right) & =1+\frac{1-C^{2}}{C^{2}} \sin ^{2}\left(C \sigma_{0}\right), \\
\tilde{g}_{\mu \nu} & =\Phi^{-1}\left(\sigma_{0}\right) g_{\mu \nu} .
\end{aligned}
$$

In the case of a stiff perfect fluid this solution corresponds to the energy density

$$
\tilde{\rho}=C^{2} \Phi^{3}\left(\sigma_{0}\right) \rho_{0} .
$$

\section{EXAMPLES OF EXACT SOLUTIONS}

As an illustration of the general techniques we consider some particular examples of exact solutions. Here we set $\Lambda=0$.

\section{A. Homogeneous cosmological solutions}

Our first examples are homogeneous isotropic cosmological solutions with an irrotational stiff perfect fluid. Consider the metric

$$
d s_{0}^{2}=-d t^{2}+t^{2 / 3}\left(d x^{2}+d y^{2}+d z^{2}\right) .
$$

This is a flat Friedman-Robertson-Walker metric for stiff perfect fluid. The fluid energy density is $8 \pi G_{*} \rho_{0}=$ $1 / 3 t^{2}$ and the 4 -velocity is $u_{\mu}=-\partial_{\mu} t$. The fluid velocity potential is $\sigma_{0}=-(1 / \sqrt{3}) \ln (t)$.

Since our seed solution is fixed the generated solutions will be completely determined only by the explicit form of $\Phi\left(\sigma_{0}\right)$. That is why, for brevity, we shall present only the explicit form of $\Phi\left(\sigma_{0}\right)$ and the energy density.

\section{Barker's theory}

Using the solution generating procedure for Barker's theory we obtain

$$
\begin{aligned}
& \Phi^{-1}(t)=1-\sin ^{2}(\beta) \cos ^{2}\left(\frac{1}{\sqrt{3}} \ln (t)\right), \\
& 8 \pi G_{*} \tilde{\rho}=\frac{\cos ^{2}(\beta)}{3 t^{2}} \Phi^{3}(t) .
\end{aligned}
$$

\section{Brans-Dicke theory}

In the case of Brans-Dicke theory the transformations (31) yield the following solution:

$$
\begin{aligned}
\Phi^{-1 / 2}(t) & =C^{2} t^{\alpha / \sqrt{3}}+D^{2} t^{-\alpha / \sqrt{3}}, \\
\tilde{\rho} & =\frac{4 C^{2} D^{2}}{3 t^{2}} \Phi^{3}(t) .
\end{aligned}
$$

$$
\text { 3. } \mathcal{A}^{2}(\varphi)=1 /\left(1+\varphi^{2}\right) \text {-theory }
$$

Solution generating formulas (53) and (54) give

$$
\begin{aligned}
\Phi(t) & =1+\frac{1-C^{2}}{C^{2}} \sin ^{2}\left(\frac{C}{\sqrt{3}} \ln (t)\right), \\
8 \pi G_{*} \tilde{\rho} & =\frac{C^{2}}{3 t^{2}} \Phi^{3}(t) .
\end{aligned}
$$

The solution (57) (presented in coordinates different from those used here) was derived by Gurevich et al 48] and by Mimoso and Wands [14 using a completely different method. Although, the solutions (56) and (58) can be also derived by the methods developed in Refs. [13], [14. they have not been presented so far in an explicit form. It is worth noting that the methods used here to derive the homogeneous cosmological solutions are much more elegant and powerful than the methods developed in the previous works on the subject.

\section{B. Inhomogeneous cosmological solutions}

Our next examples are much more interesting. We present new inhomogeneous stiff perfect fluid cosmological solutions with everywhere regular curvature invariants. The gravitational scalar and the energy density are everywhere regular, too.

We consider the following solution to the Einstein equations with an irrotational stiff perfect fluid [46], [47]:

$$
\begin{gathered}
d s_{0}^{2}=\cosh ^{2(1-b)}(2 m t) \cosh ^{4 b(2 b-1)}(m r)\left(-d t^{2}+d r^{2}\right) \\
+\cosh ^{2(1-b)}(2 m t) \sinh ^{2}(m r) \cosh ^{2(1-2 b)}(m r) d \phi^{2} \\
+\cosh ^{2 b}(2 m t) \cosh ^{4 b}(m r) d z^{2} \\
\sigma_{0}(t)=\sqrt{b^{2}-1} \arctan [\sinh (2 m t)] .
\end{gathered}
$$

The energy density and the four-velocity are given by

$$
\begin{gathered}
8 \pi G_{*} \rho_{0}= \\
4 m^{2}\left(b^{2}-1\right) \cosh ^{-2(2-b)}(2 m t) \cosh ^{-4 b(2 b-1)}(m r), \\
u_{\mu}^{(0)}=\cosh ^{1-b}(2 m t) \cosh ^{2 b(2 b-1)}(m r) .
\end{gathered}
$$

Here, $m$ and $b\left(b^{2}>1\right)$ are free parameters.

The solution is everywhere regular and satisfies the global hyperbolicity condition. The curvature invariants are regular for whole spacetime. In addition, the spacetime describing by this solution admits two commuting spacelike Killing vectors $\frac{\partial}{\partial z}$ and $\frac{\partial}{\partial \phi}$ which are mutually and hypersurface orthogonal. 


\section{Barker's theory}

Using as a seed solution (59) and applying the solution generating formulas (22) we obtain the following Barker's theory three parametric solution:

$\Phi^{-1}(t)=1-\sin ^{2}(\beta) \cos ^{2}\left(\sqrt{b^{2}-1} \arctan (\sinh (2 m t))\right)$,

$$
\tilde{\rho}=\cos ^{2}(\beta) \Phi^{3}(t) \rho_{0} .
$$

When $\cos (\beta)=0$ the solution becomes a vacuum scalar-tensor solution. That is why we shall focus our attention on the more interesting case $\cos (\beta) \neq 0$. In this case the gravitational scalar and energy density are everywhere regular in space-time. Moreover, we have calculated the curvature invariants

$$
\tilde{I}_{1}=\tilde{C}_{\mu \nu \alpha \beta} \tilde{C}^{\mu \nu \alpha \beta}, \tilde{I}_{2}=\tilde{R}_{\mu \nu} \tilde{R}^{\mu \nu}, \tilde{I}_{3}=\tilde{R}^{2}
$$

with respect to the metric $\tilde{g}_{\mu \nu}=\Phi^{-1}\left(\sigma_{0}\right) g_{\mu \nu}$ and found that they are everywhere regular. In this sense the solution (62) is nonsingular in the case $\cos (\beta) \neq 0$.

\section{Brans-Dicke theory}

In the case of Brans-Dicke theory, the solution generating formulas (31) give

$$
\begin{gathered}
\Phi^{-1 / 2}(t)=\cos ^{2}(\beta / 2) \exp \left(\alpha \sqrt{b^{2}-1} \arctan (\sinh (2 m t))\right) \\
+\sin ^{2}(\beta / 2) \exp \left(-\alpha \sqrt{b^{2}-1} \arctan (\sinh (2 m t))\right) \\
\tilde{\rho}=\sin ^{2}(\beta) \Phi^{3}(t) \rho_{0} .
\end{gathered}
$$

In the solution $(63)$ we have restricted ourselves to the subgroup $S O(2) \subset S L(2, R)$.

The gravitational scalar and the energy density are everywhere regular in space-time. The curvature invariants $\tilde{I}_{1}, \tilde{I}_{2}$ and $\tilde{I}_{3}$ are also everywhere regular. In this sense the space-time described by the solution (63) is nonsingular.

$$
\text { 3. } \mathcal{A}^{2}(\varphi)=1 /\left(1+\varphi^{2}\right) \text {-theory }
$$

Solution generating formulas (53) and (54) applied to the seed solution (59) yield the following solution:

$$
\begin{gathered}
\Phi(t)=1+\frac{1-C^{2}}{C^{2}} \sin ^{2}\left[C \sqrt{b^{2}-1} \arctan (\sinh (2 m t))\right] \\
\tilde{\rho}=C^{2} \Phi^{3}(t) \rho_{0} .
\end{gathered}
$$

The solution (64) is nonsingular. The gravitational scalar and the energy density and the curvature invariants $\tilde{I}_{1}, \tilde{I}_{2}, \tilde{I}_{3}$ are regular everywhere.

Clearly the solution generating techniques developed in the present paper allow us to construct inhomogeneous cosmological solutions with everywhere regular curvature invariants in many other scalar-tensor theories different from those considered above.

\section{Static spherically symmetric solutions}

Finally we present examples of new static, spherically symmetric solutions.

As a seed solution we take the Janis-Newman-Winicour solution 49 to the EMCSF equations:

$$
\begin{aligned}
d s_{0}^{2} & =-f_{0}^{2 \lambda} d t^{2}+f_{0}^{-2 \lambda} d r^{2}+f_{0}^{2(1-\lambda)} r^{2}\left(d \theta^{2}+\sin ^{2} \theta d \phi^{2}\right) \\
\sigma_{0} & =\sqrt{1-\lambda^{2}} \ln \left(f_{0}\right)
\end{aligned}
$$

where $f_{0}^{2}=1-\frac{2 M}{r}$ and $0 \leq \lambda \leq 1$.

When we have asymptotically flat seed backgrounds it is natural to consider the solution generating transformations preserving the asymptotic flatness of the field configuration. It is not difficult to see that the subgroup consisting of matrices of the form

$$
U=\mathcal{D}\left(\frac{\pi}{2}\right) \mathcal{O}(\beta) \mathcal{D}^{-1}\left(\frac{\pi}{2}\right)
$$

(i.e., isomorphic to $S O(2) \subset S U(2))$ preserves the asymptotics in the case of Barker's theory. In the case of Brans-Dicke theory, the subgroup preserving the asymptotics is $S O(2) \subset S L(2, R)$ consisting of the matrices $\mathcal{O}(\beta)$.

\section{Barker's theory}

The solution generating transformations which preserve the asymptotics yield the following solution:

$$
\begin{array}{r}
\Phi^{-1}\left(f_{0}\right)=1-\sin ^{2}(\beta) \sin ^{2}\left(\sqrt{1-\lambda^{2}} \ln \left(f_{0}\right)\right), \\
\sigma=\arcsin \left[\frac{\cos (\beta) \sin \left(\sqrt{1-\lambda^{2}} \ln \left(f_{0}\right)\right)}{\sqrt{1-\sin ^{2}(\beta) \sin ^{2}\left(\sqrt{1-\lambda^{2}} \ln \left(f_{0}\right)\right)}}\right] .
\end{array}
$$

\section{Brans-Dicke theory}

The scalar-tensor image of (64) in the case of BransDicke theory is

$$
\begin{gathered}
\Phi^{-1 / 2}\left(f_{0}\right)=\cos ^{2}(\beta / 2) f_{0}^{\alpha \sqrt{1-\lambda^{2}}}+\sin ^{2}(\beta / 2) f_{0}^{-\alpha \sqrt{1-\lambda^{2}}} \\
\sigma=\frac{1}{2 \alpha} \sin (\beta) \frac{f_{0}^{\alpha \sqrt{1-\lambda^{2}}}-f_{0}^{-\alpha \sqrt{1-\lambda^{2}}}}{\cos ^{2}(\beta / 2) f_{0}^{\alpha \sqrt{1-\lambda^{2}}}+\sin ^{2}(\beta / 2) f_{0}^{-\alpha \sqrt{1-\lambda^{2}}}} .
\end{gathered}
$$




$$
\text { 3. } \mathcal{A}^{2}(\varphi)=1 /\left(1+\varphi^{2}\right) \text {-theory }
$$

The solution generating transformations for the case under consideration yield the solution:

$$
\begin{aligned}
\Phi & =1+\frac{1-C^{2}}{C^{2}} \sin ^{2}\left(C \sqrt{1-\lambda^{2}} \ln \left(f_{0}\right)\right), \\
\sigma & =\frac{1+C^{2}}{2 C} \sqrt{1-\lambda^{2}} \ln \left(f_{0}\right) \\
& -\frac{1-C^{2}}{4 C^{2}} \sin \left(2 C \sqrt{1-\lambda^{2}} \ln \left(f_{0}\right)\right) .
\end{aligned}
$$

We recall that the Jordan frame metric in the above three examples is given by $\tilde{g}_{\mu \nu}=\Phi^{-1}\left(f_{0}\right) g_{\mu \nu}$ where the explicit form of $\Phi\left(f_{0}\right)$ depends on the particular scalartensor theory.

In this section we have constructed different kinds of exact scalar-tensor solutions in the presence of a stiff perfect fluid or a minimally coupled scalar field. Using the solution generating methods developed in the present work we can, of course, generate many more and much more complicated scalar-tensor solutions taking as a seed solutions the known in the literature solutions of EMCSF equations (for example see 30- 350). The physical properties of every generated class scalar-tensor solutions, however, require a separate investigation.

\section{CONCLUSION}

In this paper we have presented a general method for generating exact solutions in some scalar-tensor theories of gravity with a MCSF or irrotational stiff perfect fluid and with a potential term of a special kind for the gravitational scalar. The method is based on the symmetries of the dilaton-matter sector in the Einstein frame. In the case of Barker's theory the dilaton-matter sector possess a group of symmetry $S U(2)$. In the case of Brans-Dicke and the theory with "conformal coupling" the dilatonmatter sector is $S L(2, R)$-symmetric.

We have described an explicit algorithm for generating exact scalar-tensor solutions starting from solutions to the EMCSF equations with a cosmological term by employing the non-linear action of the symmetry group of the dilaton-matter sector.

We have also presented a general method for generating scalar-tensor solutions with a MCSF from solutions of EMCSF equations using the geodesics of the Riemannian metric (9) in the general case, when the metric (9) does not possess nontrivial symmetries.

As an illustration of the solution generating techniques explicit exact solutions have also been constructed. In particular, we have constructed inhomogeneous cosmological solutions with everywhere regular curvature invariants.

The solution generating method can be generalized for scalar-tensor-Maxwell gravity. Taking into account that the Maxwell action (in four dimensions) is conformally invariant we can immediately write the scalar-tensorMCSF-Maxwell action in the Einstein frame

$$
\begin{gathered}
S=\frac{1}{16 \pi G_{*}} \int d^{4} x\left(R-2 g^{\mu \nu} \partial_{\mu} \varphi \partial_{\nu} \varphi\right. \\
\left.-2 A^{2}(\varphi) g^{\mu \nu} \partial_{\mu} \sigma \partial_{\nu} \sigma-F_{\mu \nu} F^{\mu \nu}-\Lambda\right)
\end{gathered}
$$

As is seen the dilaton-scalar-field sector possesses the same symmetries (when they exist) as in the case of absence of Maxwell field and they can be employed to generate new solutions with electromagnetic field from known ones. In the general case, when the metric associated with the dilaton-MCSF sector may not possess nontrivial symmetries we can construct exact scalar-tensor solutions in the presence of electromagnetic field using the geodesics of the dilaton-scalar-field sector metric.

\section{ACKNOWLEDGMENTS}

I would like to thank V. Rizov for his interest in this work and stimulating discussions.

This work was partially supported by Sofia University Grant No 459/2001.

[1] C. Brans, R. Dicke, Phys. Rev. 124, 925 (1961)

[2] P. Bergman, Int. J. Theor. Phys. 1, 25 (1968)

[3] V. Wagoner, Phys. Rev. D1, 3209 (1970)

[4] C.Will, Theory and Experiment in Gravitational Physics, Cambridge University Press, Cambridge (1993)

[5] T. Damour, G. Esposito-Farese, Class. Quant. Grav. 9, 2093 (1992).

[6] C. Will, The Confrontation between General Relativity and Experiment, Living Rev.Rel. 4, 4 (2001)

[7] T. Damour, G. Esposito-Farese, Phys. Rev. Lett. 70, 2220 (1993)

[8] T. Damour, G. Esposito-Farese, Phys. Rev. D 54, 1474 (1996)

[9] D. La, P. Steinhardt, Phys. Rev. Lett. 62, 376 (1989)

[10] L. Pimentel, J. Stein-Schabes, Phys. Lett. B 216, 25 (1989)

[11] J. Barrow, K. Maeda, Nucl. Phys. B 341, 294 (1990)

[12] J. Barrow, Phys. Rev. D 47, 5329 (1993)

[13] J. Barrow, J. Mimoso, Phys. Rev. D 50, 3746 (1994)

[14] J. Mimoso, D. Wands, Phys. Rev. D 51, 477 (1995)

[15] J. Mimoso, D. Wands, Phys. Rev. D 52, 5612 (1995)

[16] J. Barrow, P. Parsons , Phys. Rev. D 55, 1906 (1997)

[17] A. Billyard , A. Coley, Mod. Phys. Letters A 12, 2121 (1997)

[18] S. Kolitch, D. Eardley, Ann. Phys. (N.Y.) 241, 128 (1995)

[19] D. Holden, D. Wands, 1998, gr-qc/9803021

[20] A. Coley Gen. Rel. Grav. 31, 1295 (1999) 
[21] J. Levin, K. Freese, Nucl. Phys. B 421, 635 (1994)

[22] C. Will, P. Steinhardt, Phys.Rev. D 52, 628 (1995)

[23] O. Bertolami, P. Martins, Phys. Rev. D 61, 064007-1 (2000)

[24] P. Fiziev, Mod. Phys. Lett. A 15, 1977 (2000)

[25] N. Bartolo, M. Pietroni, Phys. Rev. D 61, 023518 (2000)

[26] G. Esposito-Farese, D. Polarski, Phys. Rev. D 63, 063504 (2001)

[27] J. Morris, Class.Quant.Grav. 182977 (2001)

[28] Ch. Charach, Phys. Rev. D 19, 3516 (1979)

[29] Ch. Charach, S. Malin , Phys. Rev. D 21, 3284 (1980)

[30] M. Carmeli, Ch. Charach, S. Malin, Phys. Rep. 76 (1981)

[31] J. Aguirregabiria, A. Feinstein, J. Ibanez, Phys. Rev. D 48, 4662 (1993)

[32] J. Aguirregabiria, A. Feinstein, J. Ibanez, Phys. Rev. D 48, 4669 (1993)

[33] A. Feinstein, J. Ibanez, P. Labraga, J. Math. Phys. 36, 4962 (1995)

[34] A. Feinstein, R. Lazkoz, M. Vazquez-Mozo, Phys. Rev D 56, 5166 (1997)

[35] R. Lazkoz, Phys. Rev D 60, 104008 (1999)

[36] R. Tabensky, A. Taub, Commun. Math. Phys. 29, 61 (1973)

[37] J. Wainright, W. Ince, B. Marshman, Gen. Relativ. Gravit. 10, 259 (1979)

[38] K. Virbhadra, D. Narisimha ,M. Chitre, Astron. Astrophys. 337, 1 (1998)

[39] T. Matos, R. Becerril, Qlass. Quant. Grav. 18, 2015 (2001)

[40] S. Guzman, T. Matos, H. Villegas, Astron. Nachr. 320, 97 (1999)

[41] Y. Cho, Y. Keum, Qlass. Quant. Grav. 15, 907 (1998)

[42] T. Matos, A. Urena-Lopez, Phys. Rev. D62, 081302(R) (2000)

[43] N. Banerjee, D. Pavon, Class.Quant.Grav. 18, 593 (2001)

[44] B. Barker, Astrophys. J. 219, 5 (1978)

[45] J. Lidsey , D. Wands, E. Copeland, Phys. Rep. 337, 343 (2000)

[46] L. Patel, N. Dadhich, Singularity Free Inhomogeneous Cosmological Stiff Fluid Models, IUCAA preprint IUCAA- 1/93, gr-qc/9302001

[47] M. Giovannini, Phys. Rev. D57, 7223 (1998)

[48] L. Gurevich, A. Finkelstein, V. Ruban, Astrophys. Space Sci. 22, 231 (1973)

[49] I. Janis, T. Newman, J. Winicour, Phys. Rev. Lett. 20, 878 (1968) 